\title{
Visceral Adipose Tissue Promotes Pressure- Induced Heart Failure Associated with Circulating Fatty Acids
}

\section{Wenjiao Shi}

Shanghai Jiaotong University School of Medicine Xinhua Hospital

\section{Yuanzhi Peng}

Shanghai Jiaotong University School of Medicine Xinhua Hospital

Jianer Du

Shanghai Jiaotong University School of Medicine Xinhua Hospital

Xiaojian Weng ( $\nabla$ wengxiaojian@xinhuamed.com.cn )

Shanghai Jiaotong University School of Medicine Xinhua Hospital

\section{Research}

Keywords: Heart failure, visceral adipose tissue, arachidic acid, behenic acid, lignoceric acid, and docosapentaenoic acid

Posted Date: September 23rd, 2020

DOI: https://doi.org/10.21203/rs.3.rs-76236/v1

License: (c) (i) This work is licensed under a Creative Commons Attribution 4.0 International License. Read Full License 


\section{Abstract}

Background: Visceral adipose tissue (VAT) is the main source of circulating fatty acids (FAs) that provides the energy substrate for the heart. Till now, studies have not shown a clear association between individual circulating FAs and heart failure (HF). In this study, we aimed to investigate the changes in circulating FAs in HF mice and their association with VAT by removing epididymal white adipose tissue (eWAT).

Methods: Male C57BL/6J mice were divided into normal control and eWAT excision groups. Each group was then divided into sham group and HF group. The transverse aortic constriction (TAC) was used to induce pressure-mediated cardiac failure. HE staining and Masson' staining were used to observe cardiac morphology. Echocardiography was used to determine diastolic left ventricular posterior wall (LVPWd), diastolic left ventricular internal diameter (LVIDd) and fractional shortening (FS\%). RT-PCR was used to determine mRNA expression of atrial natriuretic peptide $(A N P)$, brain natriuretic peptide $(B N P), M Y H 7$, $C T G F$, collagen 1 (COL1), COL3A1 in heart tissue of mice. HPLC measurement was used to determine serum level of FAs.

Results: Cardiac enlargement and fibrosis were observed after TAC surgery evidenced by increased LVPWd, LVIDd, and increased mRNA expression of ANP, BNP, MYH7, CTGF, COL 1, COL3A1 in heart tissue $(P<0.05)$. Among various $F A s$ species, serum arachidic acid $(P<0.001)$, behenic acid $(P<0.001)$, lignoceric acid $(P<0.01)$, and docosapentaenoic acid $(P<0.05)$ levels in HF mice were significantly decreased versus sham group. However, above mentioned four serum fatty acids were significantly decreased in both sham and HF group with eWAT excision compared with eWAT reserved. In addition, the changes accompanied by LVPWd significantly decreased and FS\% significantly increased.

Conclusion: Serum arachidic acid, behenic acid, lignoceric acid, and docosapentaenoic acid levels were significantly decreased in pressure-induced HF mice, most importantly, VAT excision alleviated TACinduced cardiac failure by decreasing these four kinds of fatty acids levels.

\section{Introduction}

Heart failure (HF) is a major public health issue prevalent in approximately $2 \%$ of the population in Western countries and is associated with high morbidity and mortality rates despite pharmacological and non-pharmacological treatment [1]. Evidence suggests that development of HF is associated with abnormalities in cardiac energy metabolism [2]. The failing heart shows an energy deficit with lower levels of adenosine triphosphate (ATP) than does a normal healthy heart, probably because of altered energy substrate availability and impaired mitochondrial oxidative capacity [3]. In the healthy adult heart, the majority of ATP is produced by mitochondrial oxidation of fatty acids. Long-chain fatty acids are crucial oxidizable metabolic substrates for the heart tissue. After esterification into phospholipids, longchain fatty acids work as building blocks of cellular membranes and participate in associated signal 
pathways [4]. However, circulating long-chain FAs are heterogeneous, and the specific FAs mainly utilized in cardiomyocyte metabolism during HF development remains unknown.

Changes in the cardiac environment cause cardiac cells to release regulatory peptides which have pathophysiological roles in organs distal to the heart, such as adipose tissue, muscle, and liver, where cell death, growth, fibrosis, and remodeling are regulated $[5,6]$. Natriuretic peptides are regulatory peptides released from the heart. In addition to mediating natriuresis, diuresis, and vasodilation, natriuretic peptides enhance lipolysis and lipid mobilization $[7,8]$. Moreover, it has also been reported that these natriuretic peptides induce changes in white adipose tissue (WAT) depots promoting the development of brown adipose tissue (BAT) characteristics (such as increased thermogenesis and energy expenditure), a phenomenon termed browning or beiging [9]. These findings provide further evidence of crosstalk between the heart and the peripheral adipose tissues. VAT is not only responsible for lipid storage by lipogenesis, but also for breakdown of fatty acids (FAs) by lipolysis, consequently generating substrates for energy metabolism via $\beta$-oxidation [10]. FAs derived from VAT via lipolysis mainly serve as oxidizable substrates for the heart, and may be responsible for HF. We hypothesized that surgically removing VAT may decrease some kinds of circulating FAs levels, thus preventing HF.

In this study, we aimed to investigate the circulating long-chain fatty acids levels in pressure-induced cardiac failure models to elucidate the potential FAs mainly utilized in cardiomyocyte metabolism. In addition, we examined whether VAT excision could alleviate pressure-induced HF through changes in the levels of some FAs. This study will provide a new idea for the prevention and treatment of heart failure in the future.

\section{Materials And Methods}

\subsection{Animals}

Male C57BL/6J wild type mice were purchased from Slac Laboratory Animals (Shanghai, China), and were studied at 10 weeks of age. Animals were housed at $22^{\circ} \mathrm{C}$ with a $12-\mathrm{h}$ light/12-h dark cycle with free access to water and standard chow. The study conforms to the relevant guidelines and regulations of the Bio-X Institutes of Shanghai Jiao Tong University (Shanghai, China) and was performed after securing approval from the Animal Care and Use Committee of Shanghai Xinhua Hospital affiliated to Shanghai Jiao Tong University School of Medicine (Shanghai, China).

\subsection{Experimental models}

Ten weeks old C57BL/6J male mice (20-25 g) were divided into two groups. In one group, mice were anesthetized with 80-100 mg/kg ketamine and 5-10 mg/kg xylazine intraperitoneally, and then the epididymal white adipose tissue (eWAT) and the main visceral adipose tissue (VAT) were removed. The other group served as normal controls with eWAT reserved. Both groups were then randomly divided into sham and heart failure (HF) groups. The HF mice were established by transverse aortic constriction (TAC). Briefly, mice were anesthetized with sodium pentobarbital (50 mg/ $\mathrm{kg}$ intraperitoneally), and after 
opening the chest wall, the thoracic aorta was identified after blunt dissection through the intercostal muscles. Silk suture $(7-0)$ was placed around the transverse aorta and tied around a 26-gauge blunt needle, which was subsequently removed. Sham-operated mice underwent a similar surgical procedure without aortic constriction. After 11 weeks, cardiac structure and function were determined by echocardiography. Mice were then euthanized under isoflurane anesthesia by cervical dislocation, and the hearts were weighed and collected for further analysis.

\subsection{Echocardiography}

Echocardiography was performed 11 weeks after TAC under the guidance of M-shaped curves of twodimensional ultrasonic echocardiogram. Every 3 continuous means of the cardiac cycle were selected as the test value. Some cardiac diameters such as fractional shortening (FS), diastolic left ventricular posterior wall (LVPWd), and diastolic left ventricular internal diameter (LVIDd) were quantified as previously described [11]

\subsection{Processing of specimens}

All mice were weighed before opening the chest wall. The heart was weighed after being taken out, leaving the left ventricle intact (including the inter-ventricular septum) for further weighing. Then, the weight index of the left ventricle (weight of the left ventricle/the body weight) was calculated. Mouse serum was isolated from whole blood, and blood samples were incubated for $60 \mathrm{~min}$ at room temperature to induce coagulation. Afterwards, samples were centrifuged at $5000 \mathrm{rpm}$ for $5 \mathrm{~min}$ at $4^{\circ} \mathrm{C}$ and the supernatant (serum) was collected and stored at $-80^{\circ} \mathrm{C}$ until further analysis.

\subsection{Hematoxylin and eosin/Masson's staining}

Heart tissue samples were formalin-fixed, paraffin-embedded and stained with Hematoxylin/Eosin (HE) and Masson. Cardiac myocyte cross sectional area was assessed using HE-stained and Masson-stained sections of myocardium. After staining, the myocytes were cut into transverse sections and the perimeter of the cell borders of 50 myocytes were measured using CellSens (Olympus). Myocytes were selected in up to ten $400 x$ microscopic fields each separated into 20 equally sized squares. Only one transversally myocyte per square was measured to increase representativeness.

\subsection{Quantitative real time PCR (qRT-PCR)}

Total RNA was isolated from heart samples with TRIzol reagent (Invitrogen; Thermo Fisher Scientific, Inc.), purified with the RNEasy Kit (Qiagen, Hilden, Germany), and reverse transcribed using the oligo (dT) primers with the Transcriptor First Strand cDNA Synthesis kit (cat. no. 04896866001; Roche diagnostics, Shanghai, china), as previously described [12]. Samples were analyzed for mRNA levels using SYBRgreen Master Mix (cat. no. 04887352001; Roche diagnostics), and amplification was monitored using the CF X96 Real-Time PCR system (Bio-Rad, Munich, Germany) with the following thermocycling conditions: $5 \mathrm{~s}$ at $95^{\circ} \mathrm{C}$ followed by $30 \mathrm{~s}$ at $60^{\circ} \mathrm{C}$ for 42 cycles. The reaction mixture was composed of $10 \mu \mathrm{SYBR}$ premix Ex Taq II, $0.2 \mu \mathrm{l}$ primers, $8.8 \mu \mathrm{H}_{2} \mathrm{O}$, and $1 \mu \mathrm{l}$ DNA. Data were normalized to the expression levels of 
GAPDH (internal control) and were presented as arbitrary units normalized to wild type expression levels. Primer sequences in the present study are presented in Table 1.

Table 1

Primers used in real-time fluorescence quantitative PCR

\begin{tabular}{|c|c|c|}
\hline Gene & Forward primer $\left(5^{\prime}-3^{\prime}\right)$ & Reverse primer $\left(5^{\prime}-3^{\prime}\right)$ \\
\hline$A N P$ & AGATCTGCCCTCTTGAAAAGCA & TCGAGCAGATTTGGCTGTTATC \\
\hline$B N P$ & TCCAGAACAATCCACGATGC & GCAGCTTGAACTATGTGCCATC \\
\hline MYH7 & GCGGACATTGCCGAGTCCCAG & GCTCCAGGTCTCAGGGCTTCACA \\
\hline CTGF & GGACACGAACTCATTAGAC & TCTCACTTTGGTGGGATAG \\
\hline COL 1 & TTCTCCTGGCAAAGACGGAC & CGGCCACCATCTTGAGACTT \\
\hline COL3A1 & AGCGGCTGAGTTTTATGACG & AGCACAGGAGCAGGTGTAGA \\
\hline GAPDH & CATGACAACTTTGGCATCGT & GGATGCAGGGATGATGTTCT \\
\hline
\end{tabular}

\subsection{Analysis of fatty acids}

Mouse serum was isolated from whole blood. For FAs serum profiling, $100 \mu \mathrm{l}$ of murine serum was hydrolyzed under alkaline-methanolic conditions, neutralized and diluted in methanol (1:10) containing internal FA standards. Analysis of fatty acids was performed using HPLC (Agilent 1200 HPLC system), coupled with an Agilent 6460 triple quad mass was performed, as previously described [13]. Serum concentration of FFA was measured using a HR-NEFA kit (WAKO), as previously described [13]. Triglycerides (TG) in serum were determined using a triglyceride assay kit (DiaSys $\mathrm{GmbH}$ ), according to the manufacturer's instructions.

\subsection{Statistical analysis}

Quantitative data were expressed as mean \pm standard error of the mean (SEM). Comparison of mean values between groups was evaluated by the following tests: two-way ANOVA followed by post Bonferroni tests, two-way repeated measures ANOVA followed by post Bonferroni tests, one-way ANOVA followed by Tukey's or Bonferroni multiple comparison test, or unpaired t tests, as appropriate, and data were analyzed with GraphPad Prism software. Exact value of number is provided for each type of experiments. Statistical significance was assumed at $\mathrm{P}<0.05$. Vertical lines in the histograms indicate standard error of the mean (SEM).

\section{Results}




\subsection{Removal of eWAT attenuates TAC-induced cardiac enlargement}

To further evaluate the cardiac structure changes, mice were subjected to 11 weeks of pressure overload by transverse aortic constriction (TAC), or sham operations (Sham). The heart weights and cardiac characteristics are presented in Fig. 1. Heart weight-to-body weight ratios (HW/BW) and weight index of the left ventricle (LW/BW) were significantly increased in TAC mice compared to sham mice $(P<0.01)$, however, the ratios were significantly decreased in TAC mice with eWAT excision compared to TAC mice with eWAT reserved $(P<0.05)$. Echocardiographic measurements revealed that LVPWd and LVIDd were significantly increased $(P<0.05)$ while $F S \%$ was notably decreased $(P<0.01)$ in TAC mice compared to sham mice. However, LVPWd showed a more pronounced decrease in eWAT excised mice compared to eWAT reserved mice following TAC $(P<0.05)$. LVIDd diameters were not significantly altered between eWAT excision and eWAT reserved mice undergoing TAC. In addition, FS\% was significantly increased in TAC mice with eWAT reserved compared to eWAT excised $(P<0.05)$. These data showed that there was certain improvement in myocardial hypertrophy and cardiac efficiency after the intervention.

\subsection{Removal of eWAT changes cardiac morphology in TAC- induced HF mice}

The cardiac morphological changes are presented in Fig. 2. The size of the hearts increased markedly in size and weight compared to sham group at 11 weeks after TAC. TAC-induced cardiac enlargement was attenuated after eWAT excision. Accordingly, the myocardial area in cardiac cross section increased significantly in TAC-induced mice whereas this effect was diminished after eWAT excision. Cardiac fibrosis was significantly induced during pressure overload in HF mice as shown by Masson staining. In the TAC group, myocyte hypertrophy and fiber derangement were found with a significant amount of collagen piling in the intercellular space. The above changes were effectively alleviated after eWAT removal.

\subsection{Removal of eWAT decreases mRNA expression of ANP, COL1, and COL3A1 in pressure-induced HF mice}

In keeping with the morphological changes described above, marker genes for cardiac failure such as the $A N P, B N P, M Y H 7, C T G F, C O L 1$, and COL3A1were markedly induced by TAC in WT-hearts (Fig. 3). Cardiac hypertrophy was significantly induced during pressure overload in HF-mice as shown by augmented mRNA expression of $A N P(\mathrm{P}<0.01), B N P(\mathrm{P}<0.01)$, and $M Y H 7(\mathrm{P}<0.01)$. Cardiac fibrosis was significantly induced during pressure overload in HF-mice as shown by augmented mRNA expression of the fibrotic marker genes CTGF $(\mathrm{P}<0.01), \operatorname{COL} 1(\mathrm{P}<0.01)$ and COL3A1 $(\mathrm{P}<0.01)$. However, compared to TAC-induced HF mice with eWAT reserve, we observed a significant decrease in mRNA levels of $A N P(\mathrm{P}<$ $0.01), C O L 1(P<0.05)$ and COL3A1 $(\mathrm{P}<0.05)$ by TAC in eWAT excision mice. In addition, we found moderate decrease in mRNA levels of BNP, MYH7, and CTGF in eWAT excision mice induced by TAC, 
whereas the result was not statistically significant. Together, these data show that deletion of eWAT protects the heart against pressure-induced cardiac hypertrophy and fibrosis.

\subsection{Removal of eWAT attenuates circulating arachidic acid, behenic acid, lignoceric acid, and docosapentaenoic acid levels in pressure-induced HF mice}

To recognize potential FAs involved in the regulation of cardiac function in pressure-induced HF mice, we examined 24 kinds of FAs, triglycerides (TGs), and free circulating FAs (FFA) in serum. As depicted in TAC and sham groups presented in Fig. 4, in eWAT reserve mice, pressure overload resulted in a significant reduction of selected circulating FAs including arachidic acid $(P<0.001)$, behenic acid $(P<0.001)$, lignoceric acid $(P<0.01)$, and docosapentaenoic acid $(P<0.05)$ after TAC (Fig. 4K, R, V, U). The serum TG level was significantly increased $(P<0.05)$. In addition, the serum FFA levels were not different between the two groups. Next, we measured whether the lack of eWAT regulated the release of these preferential FAs in a selective manner. We found that in sham and TAC groups, eWAT excision significantly attenuated the circulating arachidic acid, behenic acid, lignoceric acid, and docosapentaenoic acid levels compared to eWAT reserve groups $(P<0.05)$. But the serum TG and FFA were not different between the two groups. Most importantly, after excision of eWAT, the circulating arachidic acid, behenic acid, lignoceric acid, and docosapentaenoic acid levels were not significantly different between the sham and TAC groups. Taken together, pressure-induced cardiac damage was accompanied by changes in circulating FAs among which arachidic acid, behenic acid, lignoceric acid, and docosapentaenoic acid displayed the most apparent variation, and these changes can be reversed by deletion of eWAT.

\section{Discussion}

In this study, we aimed to investigate the serum FAs in pressure-induced HF mice with eWAT reserved and eWAT excised. Mice without aortic constriction that underwent a similar surgical procedure served as controls. We demonstrated for the first time that from among multitudinous serum FAs, arachidic acid, behenic acid, lignoceric acid, and docosapentaenoic acid levels were significantly decreased in pressureinduced cardiac failure model. In addition, removal of eWAT significantly alleviated cardiac structure and function in HF mice accompanied by a decrease in these four serum FAs levels, indicating non-cardiac tissues such as eWAT participate in the regulation of cardiac failure.

The role of WAT in HF development and cardiac metabolism has been previously studied, with most studies focusing mainly on cardiac lipase function $[14,15]$. In this study, we concentrated on VAT and the impact of adipose tissue lipolysis on the development of pressure-induced cardiac failure. This study revealed that the serum triacylglycerol (TG) level was significantly increased in pressure-induced HF mice compared to controls. Our findings corroborated previous studies that reported a positive association between increased adipose tissue lipolysis and impairment of cardiac function in heart failure models $[16,17]$. Circulating FAs are liberated from WAT via hydrolysis of TG which is catalyzed by two major adipose tissue lipases, hormone-sensitive lipase (HSL) and adipose triglyceride lipase (ATGL), under hormonal control [18]. Deletion of ATGL in adipose tissue prevents pressure-induced LV failure by 
decreasing adipose tissue lipolysis [19]. Consistent with previous studies, our findings showed that removal of eWAT significantly alleviated pressure-induced cardiac hypertrophy and fibrosis, as evidenced by decreased mRNA expression of cardiac hypertrophy and fibrosis genes ANP, COL 1, and COL3A1.

Fatty acids are involved in post-translational modification of proteins through protein acylation and activation of protein kinases. Recent experimental findings have indicated that several cardiac genes are under the control of fatty acids $[20,21]$. However, there are multiple FAs present in circulating bloodstream, and it is not clear which of them are mainly involved in cardiac energy metabolism. We analyzed serum FAs using HPLC, which was previously utilized in cardiovascular research for the identification of new molecular co-mediator of exercise-induced cardiac hypertrophy [13]. Our study found that serum arachidic acid, behenic acid, lignoceric acid, and docosapentaenoic acid levels were significantly decreased in pressure-induced cardiac injury. Arachidic acid, behenic acid, and lignoceric acid are long-chain saturated FAs that are found in circulation. Circulating long-chain saturated FAs with 20 carbons or more are integrated biomarkers of diet and metabolism [22]. The long-chain saturated FAs are known components of ceramides that are involved in apoptosis and cardiac dysfunction [23, 24]. The heart of genetically engineered mice with reduced ceramides containing long-chain saturated FAs showed increased fibrosis, endoplasmic reticulum stress, and apoptosis [25]. Lemaitre et a/ have identified an association of higher levels of plasma phospholipid very-long-chain saturated fatty acids with lower risk of incident HF in a prospective cohort of older American adults [22]. Similar results were obtained in our study, indicating that decreased arachidic acid, behenic acid, and lignoceric acid were probably involved in pressure-induced cardiac failure. In addition, we also found that the serum level of docosapentaenoic acid, a long-chain unsaturated fatty acid, was significantly decreased in pressure-induced HF mice. In addition, we found no increased levels of FAs in serum of pressure-induced heart failure mice. This eliminated the probability that these reduced fatty acids were converted to other fatty acids. Thus, we hypothesized that these four fatty acids are involved in cardiac function impairment during pressure overload. However, this needs to be verified by analyzing lipid class in cardiac tissue through further studies.

The current study found that mice after removal of eWAT showed a marked reduction of these four fatty acids in both normal and pressure-induced HF mice compared to mice with eWAT reserved, which indicated that these four fatty acids may be principally derived from eWAT. In addition, we observed a slightly, but not significantly, decreased TG and FFA levels. eWAT serves as a main VAT, which can more easily produce FFA than subcutaneous adipose tissue (SAT) because VAT has higher expression of adrenergic receptors 1,2 , and 3 on the cell membrane [26, 27]. Surprisingly, no significant differences were found in circulating FFA and TG after eWAT removal in this study. One possible explanation for this might be that VST is mainly distributed around abdominal organs including epididymal adipose tissue, omental adipose tissue, mesenteric adipose tissue, retroperitoneal adipose tissue, perirenal adipose tissue, etc [28]. Although eWAT was removed in this study, other visceral adipose tissues may have also played a role in producing FFA. Another possible explanation might be because of the difference in the experimental period selected in this study. There are, however, other possible explanations that need to be further studied. The mechanisms underlying the differential impact of adipose tissue on cardiometabolic 
risk have started to be unraveled; the inability of the subcutaneous adipose tissue to expand in response to positive energy balance serves as an important mechanism that necessitates further studies on the subcutaneous adipose tissue.

\section{Conclusion}

Taken together, the results of this study demonstrate for the first time that serum arachidic acid, behenic acid, lignoceric acid, and docosapentaenoic acid levels are significantly decreased in pressure-induced heart failure. Furthermore, we showed that the decreased levels of these four FAs can be significantly reversed by removing eWAT from mice before inducing HF. However, whether these four fatty acids accumulate in cardiomyocytes remains unknown. Further research should be carried out to explore the accumulation of these fatty acids in cardiomyocytes and the damage to the mitochondrial function of cardiomyocytes, so as to provide new therapeutic targets for clinical treatment of heart failure.

\section{Abbreviations}

VAT

visceral adipose tissue; eVAT:epididymal white adipose tissue; HF:heart failure; FAs:fatty acids; LVPWd:left ventricular posterior wall; LVIDd:diastolic left ventricular internal diameter; FS\%:fractional shortening; ANP:atrial natriuretic peptide; BNP:brain natriuretic peptide.

\section{Declarations}

\section{Ethics Approval and Consent to participate}

The experimental protocol (No: XHEC-F-2017-076) was approved by Ethics Committee of Xinhua Hospital Affiliated to Shanghai Jiao Tong University School of Medicine (Shanghai, China). The animal care and experimental procedures followed the guidelines of the Bio- $X$ Institutes of Shanghai Jiao Tong University (Shanghai, China)

\section{Consent for publication}

Not applicable.

\section{Availability of data and materials}

The data used to support the findings of this study are available with the corresponding author on reasonable request.

\section{Competing interests}

The authors declare that there are no conflicts of interest. 


\section{Funding}

This work was supported by the National Natural Science Foundation of China (grant number 81901990).

\section{Authors' contributions}

Xiaojian Weng and Jianer Du designed the study. Wenjiao Shi and Yuanzhi Peng conducted the animal study and were responsible for data acquisition. Wenjiao Shi drafted the manuscript. Yuanzhi Peng critically reviewed the manuscript draft. Xiaojian Weng finalized the manuscript. All authors read and approved the content of the submitted version of the manuscript

\section{Acknowledgement}

The authors would like to thank all the colleagues who contributed to this study. This study was supported by a grant from the National Natural Science Foundation of China (grant number 81901990).

\section{References}

1. Ponikowski P, Voors AA, Anker SD, et al. 2016 ESC Guidelines for the diagnosis and treatment of acute and chronic heart failure: The Task Force for the diagnosis and treatment of acute and chronic heart failure of the European Society of Cardiology (ESC). Developed with the special contribution of the Heart Failure Association (HFA) of the ESC. Eur J Heart Fail. 2016;18(8):891-975.

2. Voros G, Ector J, Garweg C, et al. Increased Cardiac Uptake of Ketone Bodies and Free Fatty Acids in Human Heart Failure and Hypertrophic Left Ventricular Remodeling. Circ Heart Fail. 2018;11(12):e004953.

3. Conway MA, Allis J, Ouwerkerket R, et al. Detection of low phosphocreatine to ATP ratio in failing hypertrophied human myocardium by 31P magnetic resonance spectroscopy. Lancet. 1991;338(8773):973-6.

4. Vusse GJ, Bilsen M, Glatz JF. Cardiac Fatty Acid Uptake and Transport in Health and Disease. Cardiovasc Res. 2000;45(2):279-93.

5. Loncar G, Fülster S, Haehling SV, Popovic V. Metabolism and the heart: An overview of muscle, fat, and bone metabolism in heart failure. Int J Cardiol. 2013;162(2):77-85.

6. Nakamura M, Sadoshima J. Heart over mind: metabolic control of white adipose tissue and liver. Embo Molecular Medicine. 2014;6(12):1521-4.

7. Sengenès $C$, Berlan $M$, Glisezinski ID,et al. Natriuretic peptides: a new lipolytic pathway in human adipocytes. FASEB J. 2000;14(10):1345-51.

8. Moro C, Crampes F, Sengenes C, et al. Atrial natriuretic peptide contributes to physiological control of lipid mobilization in humans. FASEB J. 2004;18(7):908-10.

9. Bordicchia M, Liu D, Amri EZ, et al. Cardiac natriuretic peptides act via p38 MAPK to induce the brown fat thermogenic program in mouse and human adipocytes. J Clin Invest. 2012;122(3):1022-36. 
10. Bodis K, Roden M. Energy metabolism of white adipose tissue and insulin resistance in humans. Eur J Clin Invest. 2018;48(11):e13017.

11. Kawahara Y, Tanonaka K, Daicho T, et al. Preferable Anesthetic Conditions for Echocardiographic Determination of Murine Cardiac Function. J Pharmacol Sci. 2005;99(1):95-104.

12. Shi W, Guo ZX, Ji Y, et al. The protective effect of recombinant globular adiponectin on testis by modulating autophagy, endoplasmic reticulum stress and oxidative stress in streptozotocin-induced diabetic mice. Eur J Pharmacol. 2020;879:173132.

13. Foryst-Ludwig A, KreissI MC, Benzet V, et al. Adipose Tissue Lipolysis Promotes Exercise-induced Cardiac Hypertrophy Involving the Lipokine C16:1n7-Palmitoleate. J Biol Chem. 2015;290(39):23603-15.

14. Zierler KA, Jaeger D, Pollaket NM, et al. Functional cardiac lipolysis in mice critically depends on comparative gene identification-58. J Biol Chem. 2013;288(14):9892-904.

15. Kienesberger PC, Pulinilkunnil T, Sunget MMY, et al. Myocardial ATGL overexpression decreases the reliance on fatty acid oxidation and protects against pressure overload-induced cardiac dysfunction. Mol Cell Biol. 2012;32(4):740-50.

16. Polak J, Kotrc M, Wedellova Z, et al. Lipolytic Effects of B-Type Natriuretic Peptide1-32 in Adipose Tissue of Heart Failure Patients Compared With Healthy Controls. J Am Coll Cardiol. 2011;58(11):1119-25.

17. Lommi J, Kupari M, Yki-Järvinen H. Free Fatty Acid Kinetics and Oxidation in Congestive Heart Failure. Am J Cardiol. 1998;81(1):45-50.

18. Young SG, Zechner R. Biochemistry and pathophysiology of intravascular and intracellular lipolysis. Genes Dev. 2013;27(5):459-84.

19. Salatzki J, Foryst-Ludwig A, Bentele K, et al. Adipose tissue ATGL modifies the cardiac lipidome in pressure-overload-induced left ventricular failure. PLoS Genet. 2018;14(1):e1007171.

20. Bilsen MV, Vries JED, Vusse GJVD. Long-term effects of fatty acids on cell viability and gene expression of neonatal cardiac myocytes. Prostaglandins Leukot Essent Fatty Acids. 1997;57(1):3945.

21. Brandt JM, Djouadi F, Kelly DP. Fatty Acids Activate Transcription of the Muscle Carnitine Palmitoyltransferase I Gene in Cardiac Myocytes via the Peroxisome Proliferator-activated Receptor a. J Biol Chem. 1998;273(37):23786-92.

22. Lemaitre RN, McKnight B, Sotoodehnia N, et al. Circulating Very Long-Chain Saturated Fatty Acids and Heart Failure: The Cardiovascular Health Study. J Am Heart Assoc. 2018;7(21):e010019.

23. Ohno Y, Suto S, Yamanaka M, et al. ELOVL1 production of C24 acyl-CoAs is linked to C24 sphingolipid synthesis. Proc Natl Acad Sci U S A. 2010;107(43):18439-44.

24. Park TS, Hu Y, Noh HL, et al. Ceramide is a cardiotoxin in lipotoxic cardiomyopathy. J Lipid Res. 2008;49(10):2101-12. 
25. Lee SY, Kim JR, Hu Y, et al. Cardiomyocyte Specific Deficiency of Serine Palmitoyltransferase Subunit 2 Reduces Ceramide but Leads to Cardiac Dysfunction. J Biol Chem. 2012;287(22):18429-39.

26. Arner $P$, Hellström L, Wahrenberg $H$, Brönnegård $M$. Beta-adrenoceptor expression in human fat cells from different regions. J Clin Invest. 1990;86(5):1595-600.

27. Karpe F, Pinnick KE. Biology of upper-body and lower-body adipose tissue-link to whole-body phenotypes. Nat Rev Endocrinol. 2015;11(2):90-100.

28. Wajchenberg BL. Subcutaneous and visceral adipose tissue: their relation to the metabolic syndrome. Endocr Rev. 2000;21(6):697-738.

\section{Figures}

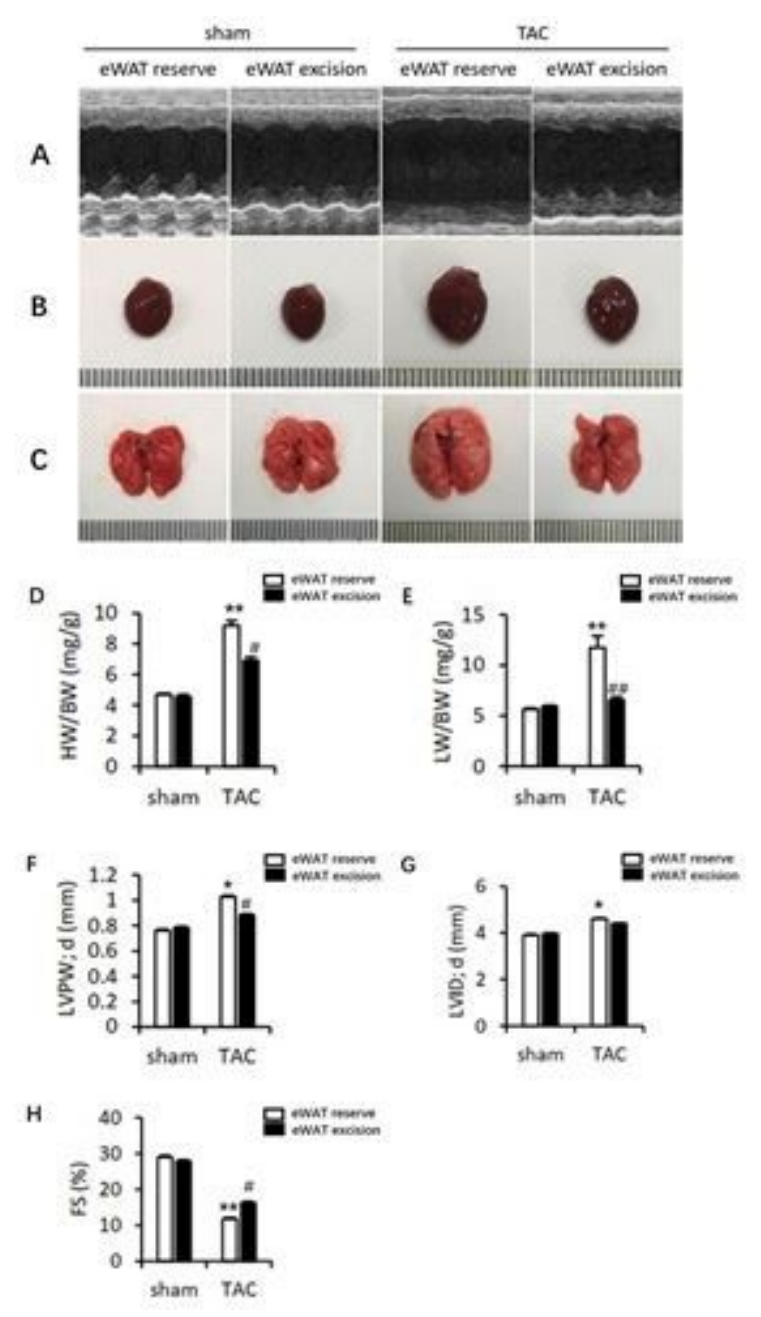

Figure 1

Changes in heart weight and cardiac structure in the four groups. A: Representative M-Mode images of the echocardiographic analysis. B and C: Representative images of the hearts. D: Heart weight (HW)/ body weight $(B W)$ ratio (mean and $S E M, n=8)$. E: Left ventricle weight $(L W) /$ body weight $(B W)$ ratio (mean and SEM, $n=8$ ). F: Left ventricular posterior wall in diastol (LVPW; $d$ ) (mean and SEM, $n=8$ ). G: Left-ventricular internal diameter in diastole (LVID; d) (mean and SEM, $n=8)$. H: Fractional shortening [\%] 
(FS) (mean and SEM, $\mathrm{n}=8$ ). sham: normal control group. TAC: transverse aortic constriction induced heart failure group. eWAT: epididymal white adipose tissue. ${ }^{*} \mathrm{P}<0.05 \mathrm{vs}$. sham + eWAT reserve, ${ }^{*} \mathrm{P}<0.01$ vs. sham + eWAT reserve, $\# \mathrm{P}<0.05$ vs. TAC + eWAT reserve, \#\#P<0.01 vs. TAC + eWAT reserve; two-way ANOVA (Bonferroni post-test).

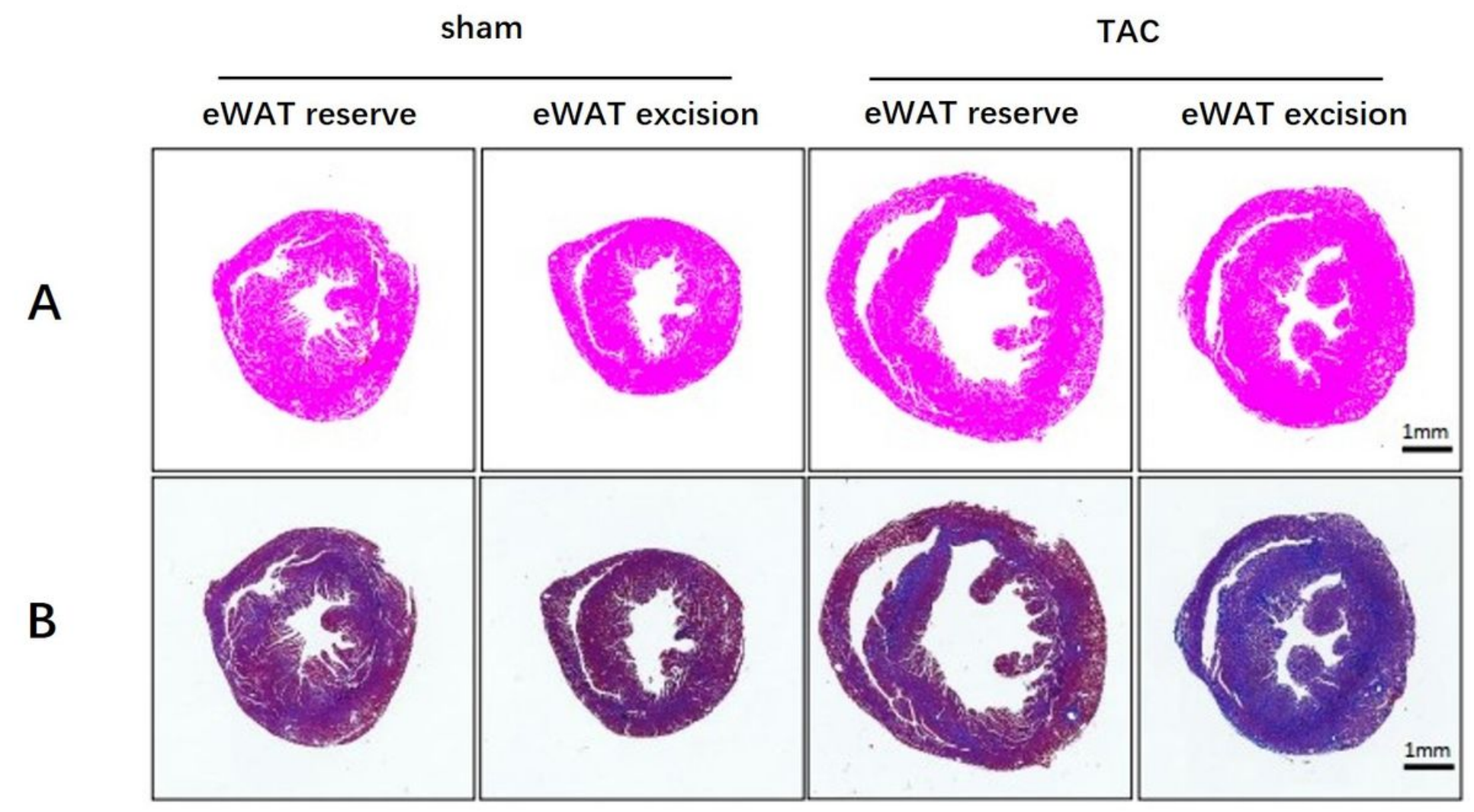

Figure 2

Cardiac morphological changes in the four groups. A: Representative microscopic cross-sections of the hearts stained with hematoxylin/ eosin (H/E). B: Representative microscopic cross-sections of the hearts stained with Masson. sham: normal control group. TAC: transverse aortic constriction induced heart failure group. eWAT: epididymal white adipose tissue. 

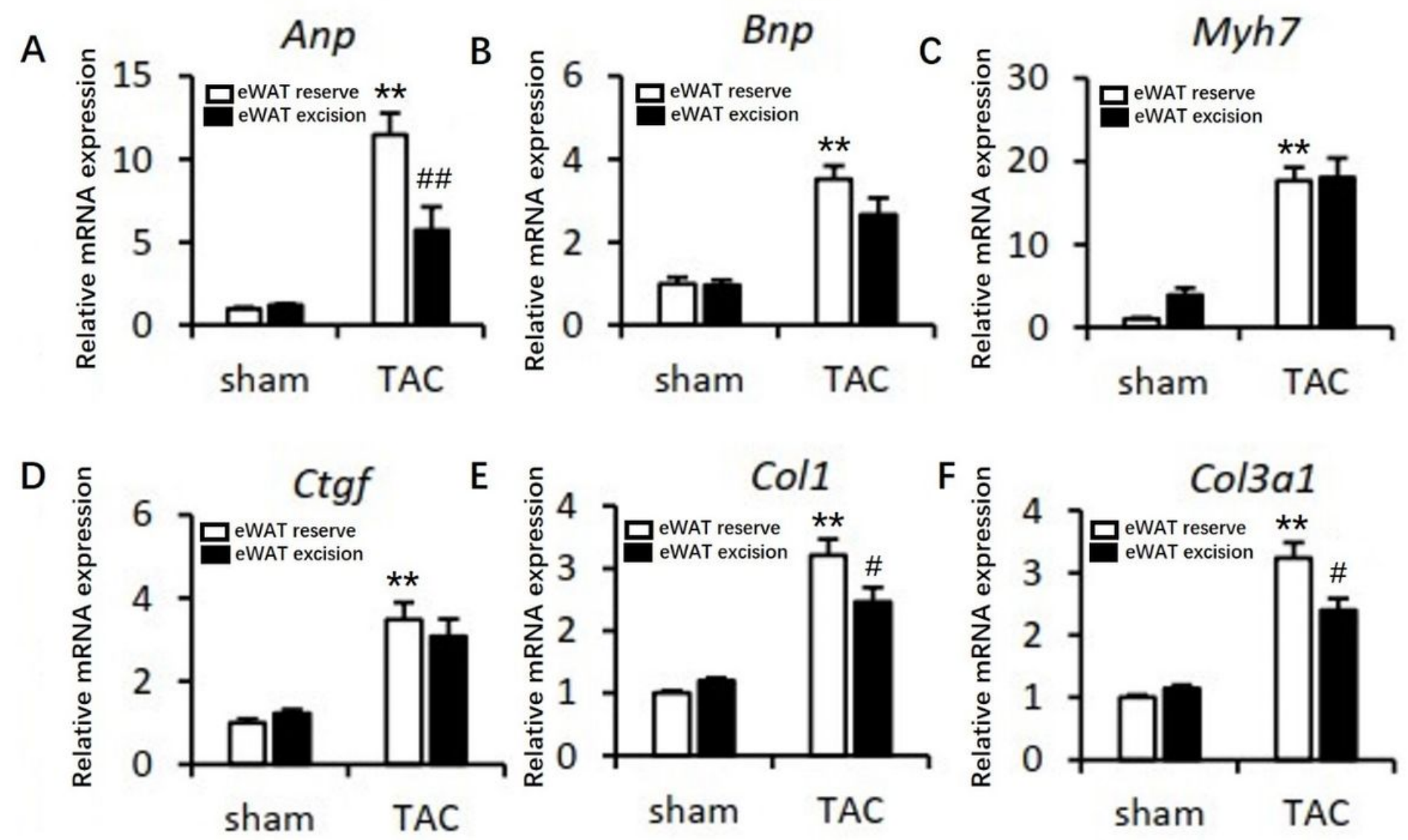

Figure 3

Removal of eWAT decreases mRNA expression of ANP, COL1, and COL3A1 in TAC-induced HF mice. The mRNA expression of ANP, BNP, MYH7, CTGF, COL1, and COL3A1 were detected by real-time fluorescence quantitative polymerase chain reaction (RT-PCR) analysis. Relative gene expression levels were normalized to that of GAPDH. Data are presented as $x$-fold over sham mice (mean and SEM, $n=8$ ). Statistical significance was determined using two-way analysis of variance $\left({ }^{*} P<0.05\right.$ vs. sham + eWAT reserve, ${ }^{*} P<<0.01$ vs. sham + eWAT reserve, $\# P<0.05$ vs. TAC + eWAT reserve, $\# \# P<0.01$ vs. TAC + eWAT reserve). ANP: Atrial natriuretic peptide; BNP: Brain natriuretic peptide; sham: normal control group. TAC: transverse aortic constriction induced heart failure group. eWAT: epididymal white adipose tissue. 


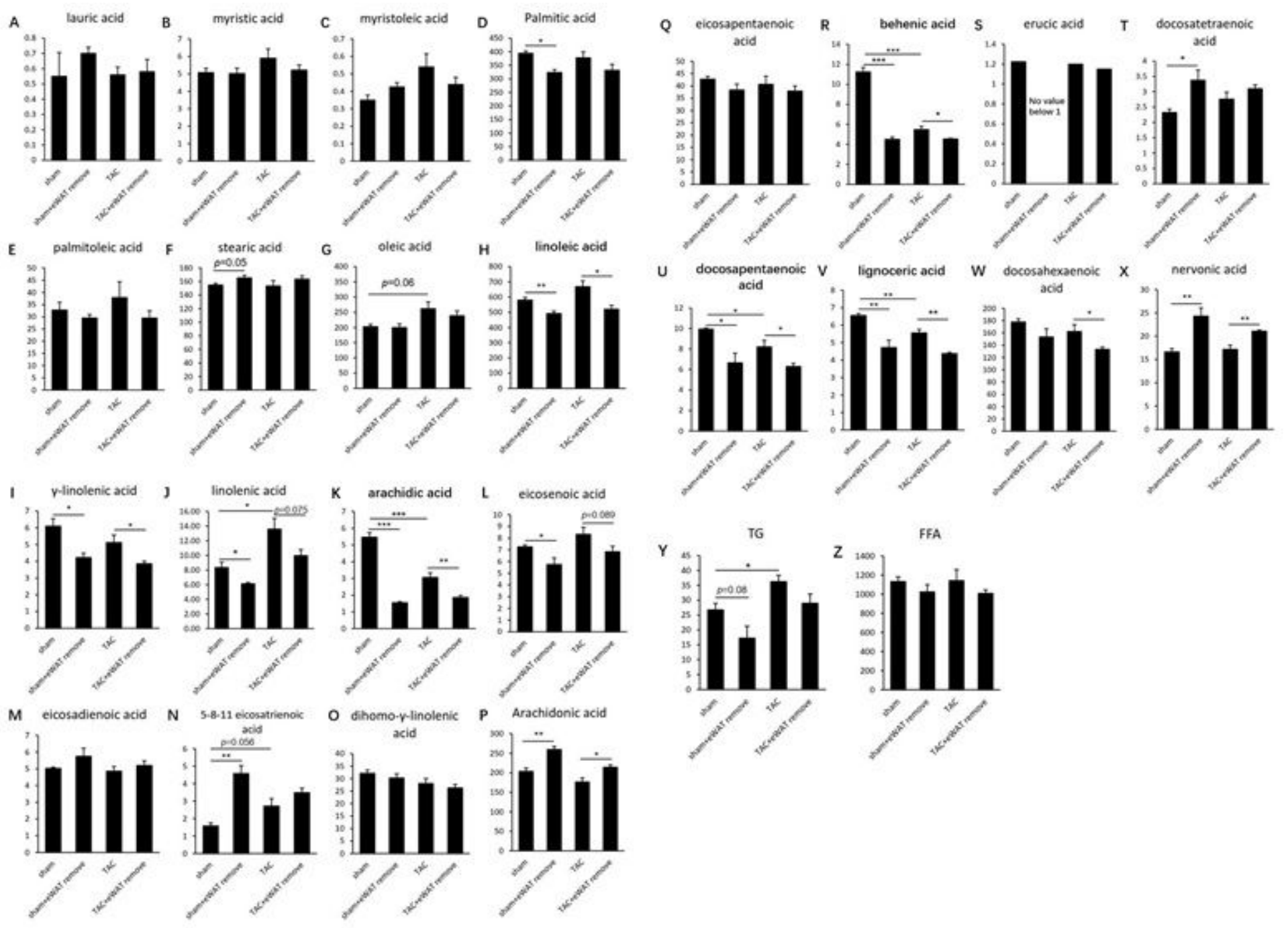

Figure 4

Removal of eWAT attenuates circulating arachidic acid, behenic acid, lignoceric acid, and docosapentaenoic acid levels. A-X: Profile of selected serum FAs analyzed by rapid resolution HPLC/Tandem MS. Y: Analysis of serum triglycerides (TG). Z: Analysis of serum free fatty acids (FFA). Data are presented as $x$-fold over sham mice (mean and SEM, $n=8$ ). Statistical significance was determined using two-way analysis of variance $\left({ }^{*} P<0.05\right.$, $\left.{ }^{*} P<0.01,{ }^{*} P<0.001\right)$. 